\title{
Battery Storage Utilization for Cost and Imbalance Reduction in a Balancing Group
}

\author{
Nikolaus Wirtz, Graduate Student Member, IEEE, and Antonello Monti, Senior Member, IEEE \\ Institute for Automation of Complex Power Systems \\ RWTH Aachen University \\ Aachen, Germany \\ nwirtz@eonerc.rwth-aachen.de, amonti@eonerc.rwth-aachen.de
}

\begin{abstract}
In this paper, the theoretical optimum of a battery utilization in a balancing group for cost reduction and energy imbalance reduction is investigated. To estimate the theoretical optimum, an offline optimization of the battery schedule is performed over a horizon of one week. Different strategies are applied, prioritizing either the cost reduction or the energy imbalance reduction objective to investigate the potential to fulfill both objectives and to determine the trade-off between them. The investigation of the scaling effects of increasing the number of batteries highlights the advantages and disadvantages of the applied strategies. Finally, it is shown that batteries can be utilized to fullfill both objectives to a certain degree, but the potential is limited due to diminishing marginal benefit when increasing the number of batteries.
\end{abstract}

Index Terms-Battery Storage System, Energy Market, Balancing Group Management

\section{INTRODUCTION}

The shift to renewable energy sources causes an increasing need and potential for new applications for energy storage in the electric power system. Battery Energy Storage Systems (BESS) are used both in mobile and stationary applications, providing high rate of change of power and a modular design due to cell-based construction of batteries, which enable dynamic operational behavior and scalability in terms of power and capacity needed for the respective application. However, large-scale batteries for power system applications are still expensive and hence, for feasibility of use, it is essential to maximize the benefit they provide.

One way is the re-use of batteries formerly of electric vehicles, so called second-life batteries, in stationary applications. Even if the maximum capacity of these batteries is already decreased due to aging, it may still be sufficient for stationary uses where high energy density is not as important as for mobile applications.

(C) (C) 2018 IEEE. Personal use of this material is permitted. Permission from IEEE must be obtained for all other uses, in any current or future media, including reprinting/republishing this material for advertising or promotional purposes, creating new collective works, for resale or redistribution to servers or lists, or reuse of any copyrighted component of this work in other works.

The project Energy Local Storage Advanced system (ELSA) has received funding from the European Union's Horizon 2020 research and innovation programme under grant agreement No 646125 .
For stationary applications of energy storage systems, multiple use cases and scenarios have been investigated, often focusing on compensation of local imbalances caused by renewable generation and load forecast errors as in [1], [2]. Opposed to these low-level applications oriented towards the local physical grid, utilization of BESS for balancing group management requires a more abstract and market-oriented perspective.

In [3], a two-level strategy for balancing group optimization is proposed, providing a strategy for formation of balancing groups and an on-line storage scheduling to reduce imbalances in these balancing groups. However, it is concentrating on the Japanese market where a fixed imbalance tariff system is implemented compared to varying energy imbalances prices in the German market.

A different approach is depicted in [4], where the arbitrage between day-ahead and imbalance markets are exploited by utilization of batteries in the Dutch market.

For the German market, a concept for reducing energy imbalances in a balancing group by utilization of a virtual power plant is described in [5], yet this concept focuses only on the imbalance reduction and neglects cost effects.

This paper evaluates the utilization of batteries for the management of a balancing group as an example for stationary second-life battery use cases [6]. The theoretical optimum for cost reduction and energy imbalance reduction is determined by the optimization of a schedule for a BESS for different operation strategies. Scaling effects on the theoretical optimum are investigated by increasing the number of batteries. The estimation of the theoretical optimum and the investigation of scaling effects can be used to assess the potential benefit of the BESS utilization in balancing groups and to compare this use case to other options applicable to stationary BESS.

\section{BALANCING GROUP CONCEPT IN THE GERMAN MARKET}

To provide and secure the stability of the electric power system, careful planning of power system operation is required. For each participant - i.e. consumer or producer of electrical energy - the production and consumption are scheduled or forecasted. Traditionally, consumers have a fixed consumption schedule, either based on (industrial) plant operation or based 
on consumption assumptions, e.g. on household level. Power plants are operated accordingly to meet the demand. The responsibilities and the information flow necessary for this procedure are managed with the help of balancing groups.

A balancing group is defined as an aggregation of different participants in the electrical energy market, which may be consumers or producers of electrical energy [7]. The balancing group manager is responsible for compensating the deviations from the scheduled energy production or consumption within the balancing group by energy trading, minimizing the remainder in the schedule. Deviations may cause the need for balancing energy to be injected or absorbed in the power system, provided by generation or consumption units participating in the balancing reserve market.

The remuneration for these units are paid by the the balancing groups where deviations occur, utilizing a transfer mechanism and must be covered by the respective balancing group responsible party. The accounting of imbalances is based on the deviation from the balancing group schedule over a 15minute-period. To be completely balanced over the horizon of one day, the deviation from the schedule must be zero in 96 consecutive 15-minute-periods.

Especially in case of fluctuating renewable energy generation with schedules based on weather forecast, additional deviations from the schedule may occur. Any deviations can be compensated by additional energy trading, yet another option is the utilization of flexibility in the balancing group. Given a lead time of five to 30 minutes for continuous intra-day trades in Germany, the utilization of fast-reacting flexibilities inside a balancing group can provide an adaption to deviations from forecasts closer to delivery or even during the delivery period. These flexibilities, e.g. BESS do not need to be owned or operated by the balancing responsible party. Instead, the BESS can also be operated by a third party, as long as a quick response to control commands is guaranteed.

\section{SET-UP}

The set-up is designed to evaluate the theoretical optimum in terms of cost reduction and energy imbalance reduction of the BESS utilization to support balancing group management. The theoretical optimum is determined by performing an offline optimization of a BESS schedule using post-delivery data for the remaining balancing energy of a balancing group and the corresponding price. To evaluate scaling effects on cost reduction and energy imbalance reduction, the number of batteries is increased in steps of 25 batteries from 0 to 150 . The BESS schedule resulting from optimization contains charge or discharge power in a 15-minute-resolution.

The offline simulation is based on historical data for a real balancing group [8], consisting of time series data for balancing energy and balancing energy prices in a 15-minuteresolution, corresponding to the time resolution in German intraday trading. The horizon covers one week, therefore each time series consists of 672 values.

To achieve the theoretical optimum in an operational application, the following requirements would have to be fulfilled:
- Perfectly accurate forecast of balancing energy for the individual balancing group

- Perfectly accurate forecast of balancing energy price for the balancing energy market

- Forecasts must be available before delivery to allow for optimization to generate the BESS schedule and control batteries accordingly

- Forecasts must cover the same horizon as offline optimization

In reality, perfect forecasts are unrealistic, especially for long optimization horizons. A moving horizon may be used in a model predictive control approach to achieve better forecast accuracy and allow for corrections during operation. However, in this case the theoretical optimum as calculated for the offline simulation may not be achieved.

\section{ASSESSMENT OF THE THEORETICAL OPTIMUM}

To assess the theoretical optimum for cost reduction and energy imbalance reduction an optimization of the BESS schedule is performed. Two different objectives - both according to the goals of the balancing group management - must be considered for this optimization:

1) Minimization of costs caused by deviations from scheduled energy in a balancing group

2) Minimization of total balancing energy as defined in the balancing group agreement between balancing group responsible party and Transmission System Operator (TSO) [9], urging balancing group responsible parties to reduce total balancing energy by applying reasonable measures

These objectives are not necessarily pulling in the same direction, as at certain time periods the schedule deviation of a specific balancing group can be opposed to the schedule deviation of all balancing groups in a specific market area. This may result for example in the need to feed power into the grid to reduce imbalances, while at the same time increasing costs for the balancing group. The consequences for BESS operation are shown in table I. A positive price denotes additional costs for the balancing group when injecting additional power into the grid. A positive imbalance denotes a power excess in the balancing group, that is additional power is injected into the grid.

TABLE I

BESS OPERATION CASES

\begin{tabular}{|l|l|l|}
\hline & positive imbalance & negative imbalance \\
\hline positive price & $\begin{array}{l}\text { (1) Charge BESS to } \\
\text { reduce cost and im- } \\
\text { balance }\end{array}$ & $\begin{array}{l}\text { (2) Only costs OR } \\
\text { imbalance can be } \\
\text { reduced }\end{array}$ \\
\hline negative price & $\begin{array}{l}\text { (3) Only costs OR } \\
\text { imbalance can be } \\
\text { reduced }\end{array}$ & $\begin{array}{l}\text { BESS to reduce } \\
\text { cost and imbalance }\end{array}$ \\
\hline
\end{tabular}

As can be seen, for cases (2) and (3) the BESS operation is not obvious. To deal with these cases, different strategies will be applied and compared. 


\section{A. Optimization objectives}

A schedule for the BESS operation is obtained as output of the optimization, consisting of setpoints for charging or discharging power in a 15-minute resolution. Technical restrictions of the BESS or the power system for maximum rate of change of power are not considered in the optimization.

The cost reduction strategy aims purely at reducing costs for the balancing group and neglects the imbalance reduction objective. Using residual energy imbalance $E_{\text {Imbalance }}^{\text {residual }}(t)$ and respective price Price Imbalance $_{\text {Im }}($ ), the objective function for cost minimization is:

$$
\min . \sum_{t=0}^{T} E_{\text {Imbalance }}^{\text {residual }}(t) * \text { Price }_{\text {Imbalance }}(t)
$$

The energy imbalance reduction strategy aims purely at reducing imbalances and neglects the cost reduction objective. Objective function of imbalance minimization is given by:

$$
\min . \sum_{t=0}^{T} a b s\left(E_{\text {Imbalance }}^{\text {residual }}(t)\right)
$$

As shown in Eqn. 2, the optimization function for imbalance minimization is the sum of the absolute values of the energy imbalance over the time window from $t=0$ to $T$, as a negative deviation is equally detrimental than a positive deviation. Moreover, the imbalance must be minimized in every time period based on the 15-minute-resolution, as deviations in opposite directions in different time periods will not compensate each other. Due to the use of absolute values, the objective function is not linear. For linearization, binary method [10] was used, splitting energy imbalance $E_{\text {Imbalance }}^{\text {residual }}(t)$ in a positive and negative part:

$$
E_{\text {Imbalance }}^{\text {residual }}(t)=E_{\text {Imbalance }}^{\text {residual }}(t)+E_{\text {Imbalance }}^{\text {residual- }}(t)
$$

Introducing a binary variable

$$
Y \in\{0,1\}
$$

And using the upper boundary $U$ and the lower boundary $L$, one of the parts will be forced to zero at any time period

$$
\begin{gathered}
0<E_{\text {Imbalance }}^{\text {residual }+}(t)<U * Y \\
L *(1-Y)<E_{\text {Imbalance }}^{\text {residual }}(t)<0
\end{gathered}
$$

\section{B. Case-dependent operation}

For the case-dependent operation, the BESS will only be operated at a certain time period if cases (1) or (4) apply. In cases (2) or (3), the BESS will be blocked in the respective time period, which means the batteries are neither charged nor discharged. This operation strategy should ensure the simultaneous reduction of costs and imbalances in each time period.
The optimization objective can either be cost or imbalance minimization, using the respective objective functions given in Eqn. 1 and Eqn. 2. However, to implement blocking of the batteries in cases (2) and (3), the energy imbalance data for the examined horizon is pre-processed before performing the optimization. For each time period, case (2) or (3) applies if the product of cost and imbalance is negative. All these time periods are removed from the energy imbalance data, so the optimization is performed with a shortened horizon. As output, a schedule for the BESS operation in cases (1) and (4) is obtained. In cases (2) and (3) BESS power is fixed to zero. Combining these schedules yields the full schedule for the BESS.

\section{Multi-Objective Optimization}

Using the lexicographic method [11], multiple objectives are considered in the optimization, pre-defining the priority of the different objectives. The prioritization enables the utilization of flexibility potential, which is remaining after optimization for the primary objective, in the BESS schedule to optimize for the secondary objective. Firstly, the optimization is performed for the primary optimization objective. The results are then used as input for a second optimization using the secondary optimization objective.

In total, six different scenarios are obtained, structured as shown in table II.

TABLE II

OPTIMIZATION SCENARIOS

\begin{tabular}{|l|l|l|l|}
\hline $\begin{array}{l}\text { Multi- } \\
\text { Objective } \\
\text { Optimization }\end{array}$ & No & No & Yes \\
\hline $\begin{array}{l}\text { BESS } \\
\text { operation }\end{array}$ & $\begin{array}{l}\text { Cases (1) and } \\
\text { (4) }\end{array}$ & All cases & All cases \\
\hline $\begin{array}{l}\text { Cost objective } \\
\text { first) }\end{array}$ & Cost-posProd & Cost-simple & Cost-lexico \\
\hline $\begin{array}{l}\text { Imbalance ob- } \\
\text { jective (first) }\end{array}$ & $\begin{array}{l}\text { Imba- } \\
\text { posProd }\end{array}$ & Imba-simple & Imba-lexico \\
\hline
\end{tabular}

\section{Constraints}

For all strategies, identical constraints have been used for optimization. These are given by the BESS limitations and the relation between BESS and balancing group power. The relation of average power over a 15-minute-period is defined in Eqn. 7 for the residual imbalance power $P_{\text {imbalance }}^{\text {residual }}(t)$, with the imbalance of the balancing group $P_{\text {imbalance }}(t)$, the BESS charging power $P_{\text {charge }}(t)$ and the BESS discharging power $P_{\text {discharge }}(t)$. The binary variables $T_{\text {charge }}(t)$ and $T_{\text {discharge }}(t)$ indicate charging and discharging operation respectively, while Eqn. 8 ensures that only one of them can be equal to 1 .

$$
\begin{array}{r}
P_{\text {imbalance }}^{\text {residual }}(t)=P_{\text {imbalance }}(t) \\
+P_{\text {charge }}(t) * T_{\text {charge }}(t) \\
+P_{\text {discharge }}(t) * T_{\text {discharge }}(t)
\end{array}
$$




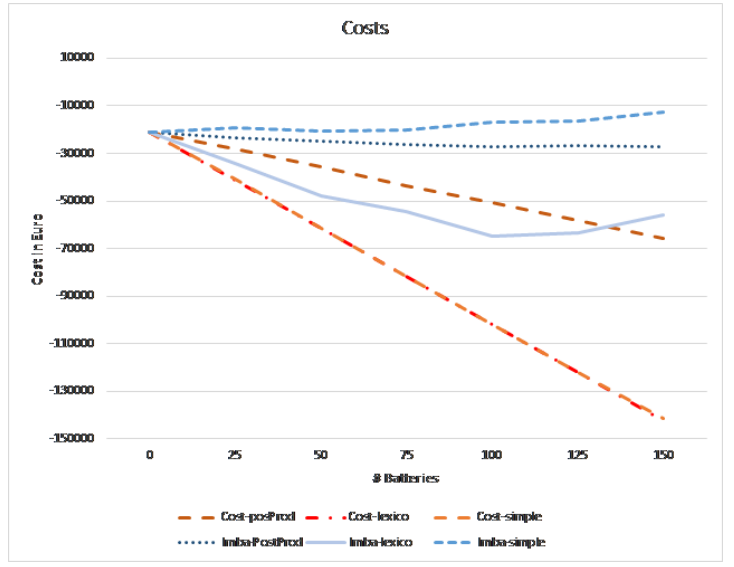

Fig. 1. Costs with battery

$$
T_{\text {charge }}(t)+T_{\text {discharge }}(t)<=1
$$

Charging and discharging efficiency $\eta_{\text {charge }}$ and $\eta_{\text {discharge }}$ of the batteries are considered in Eqn. 9, calculating the internal power of the batteries $P_{b a t}(t)$.

$$
\begin{array}{r}
P_{\text {bat }}(t)=P_{\text {charge }}(t) * T_{\text {charge }}(t) * \eta_{\text {charge }} \\
+\frac{P_{\text {discharge }}(t) * T_{\text {discharge }}(t)}{\eta_{\text {discharge }}}
\end{array}
$$

Using $P_{b a t}(t)$, the energy stored in the batteries $E_{b a t}(t)$ is calculated in Eqn. 10 and Eqn. 11 using the State of Charge (SOC) given at the beginning of the horizon $S O C_{t=0}$ and the maximum capacity of the BESS $E_{b a t}^{\max }$.

$$
\begin{gathered}
E_{b a t}(t=0)=S O C_{t=0} * E_{b a t}^{\text {max }}-P_{b a t}(t=0) * \Delta t \\
E_{\text {bat }}(t>0)=E_{\text {bat }}(t-1)-P_{b a t}(t) * \Delta t
\end{gathered}
$$

In addition, according to Eqn. 12 the SOC is the same at the beginning and end of the optimization horizon, ensuring that the restoration of the starting conditions of the BESS is not moved behind the horizon thus distorting the optimization results.

$$
S O C_{t=0}=S O C_{t=T}
$$

\section{Evaluation of POTENTIAL BENEFITS}

To evaluate the potential benefit of the battery utilization in terms of the theoretical optimum of cost reduction and energy imbalance reduction, the optimization was performed for each of the six strategies described above, varying the number of batteries from 0 to 150 . Historical data from a real balancing group was used as input for the optimization [8].

Due to the investigation of scaling effects, marginal benefits can be estimated for the utilization of additional batteries

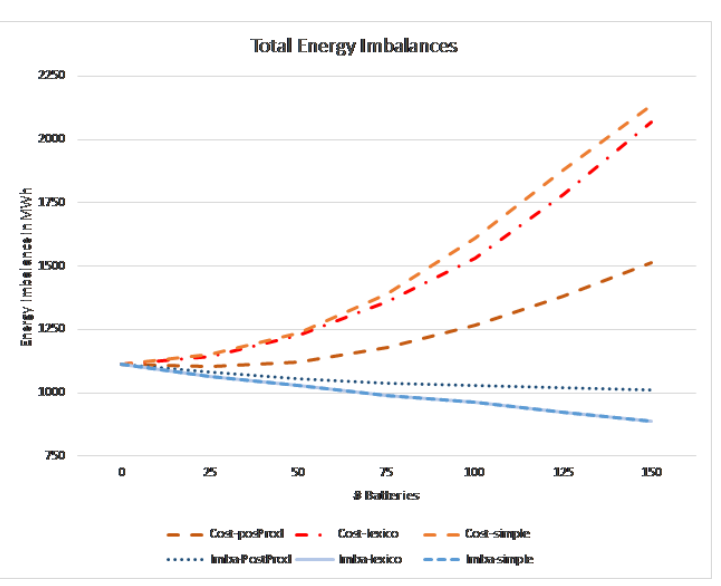

Fig. 2. Energy imbalances with battery

in regard to the optimization objectives. In the case of 0 batteries, no flexibility is available for reducing costs or energy imbalances, so this case can be seen as a reference for the different strategies. The costs are negative even in the reference case, as the existing imbalances correspond with beneficial imbalance prices for the chosen time interval of the balancing group. This effect purely results from the input data and not from the optimization or the applied strategies.

For the cost optimization, there is no lower limit, i.e. the lower the costs, the higher the earnings for the balancing group. The energy imbalance in the reference case is around $1,115 \mathrm{MWh}$ with a lower limit of 0 in the optimization, which would mean that the group is balanced in any time period.

However, the BESS utilization will result in an energy shifting to compensate positive and negative deviations in the balancing group. Achieving a full compensation is only possible if Eqn. 13, Eqn. 14 and Eqn. 15 apply, where $P_{\text {charge }}^{\text {rated }}$ is the rated BESS charging power as a positive value and $P_{\text {discharge }}^{\text {rated }}$ is the rated BESS discharging power as a negative value.

$$
\begin{gathered}
\max .\left(P_{\text {imbalance }}(t)\right)-P_{\text {charge }}^{\text {rated }}<=0 \\
\operatorname{min.}\left(P_{\text {imbalance }}(t)\right)-P_{\text {discharge }}^{\text {rated }}>=0
\end{gathered}
$$

$$
E_{\text {imbalance }}^{\text {totalpos. }} * \eta_{\text {charge }} * \eta_{\text {discharge }}=E_{\text {imbalance }}^{\text {totalneg. }}
$$

With the total positive and negative energy deviation $E_{\text {imbalance }}^{\text {totalpos. }}$ and $E_{\text {imbalance }}^{\text {totalneg. }}$ and the charging and discharging efficiency $\eta_{\text {charge }}$ and $\eta_{\text {discharge }}$. Long-term self discharge is neglected here. With this relation, the minimum imbalance that can be achieved can be calculated as

$$
\begin{array}{r}
E_{\text {imbalance }}^{\text {residualmin }}=E_{\text {imbalance }}^{\text {total }} \\
-2 * \max .\left\{E_{\text {imbalance }}^{\text {totalpos. }} * \eta_{\text {charge }} * \eta_{\text {discharge }}, E_{\text {imbalance }}^{\text {totalneg. }}\right\}
\end{array}
$$


For the input data used here, a minimal imbalance of 236 MWh is calculated, giving a potential maximal reduction of $878 \mathrm{MWh}$. As the operation time interval is shorter for scenarios Cost-posProd and Imba-posProd, a potential maximal reduction of $163 \mathrm{MWh}$ is calculated.

\section{A. Cost reduction as primary objective}

Applying cost optimization leads to a linear correlation between number of batteries and imbalance costs thus marginal benefit is not diminishing but constant. For the Cost-simple and Cost-lexico scenarios, a cost decrease of about 800 $€ /$ battery can be achieved over a horizon of one week. For the Cost-posProd scenarios, cost decrease is only $300 € /$ battery, as the batteries are not operated all the time.

In all three scenarios, the BESS operation is based on exploiting the imbalance price changes by creating higher energy imbalances, which is undesired by the TSO, thus reflected in the balancing group agreement. The total energy imbalances are increasing massively in all three scenarios, as shown in Fig. 2.

The subordinated optimization of energy imbalances in the Cost-lexico scenario causes only a little improvement compared to the Cost-simple scenario and a simultaneous minimization of costs and imbalances is not possible for these scenarios. This applies even to the Cost-posProd scenario, which was supposed to prevent this effect. The failure of this strategy to decrease imbalances is caused by an overshoot behavior, where the BESS not only charges to precisely compensate for positive imbalances, but charge with higher power than necessary, creating new, negative imbalances. The overshoot behaviour can be prevented by setting a limit for BESS charging power according to the positive imbalance in a certain time period, yet this was not implemented here. A similar effect can also be observed for discharging and can be prevented by setting a limit for BESS discharging power, respecively.

A focus on cost optimization is only beneficial from the economic perspective of the balancing group manager. From the perspective of the TSO, the behavior is not desired as higher unscheduled imbalances may cause instabilities in the power system.

\section{B. Energy imbalance reduction as primary objective}

Applying imbalance optimization leads to a decrease of energy imbalances of up to $226 \mathrm{MWh}$ or $26 \%$ of the optimum for the Imba-simple and Imba-lexico scenarios and up to 105 MWh or $12 \%$ for the Imba-posProd scenario. The correlation between number of batteries and energy imbalance is not linear for any of the scenarios, resulting in a diminishing marginal benefit for imbalance optimization.

The effects on costs is diverse for all three scenarios. As in Imba-simple scenario the imbalance costs are not considered it is not surprising that costs are even increasing there. ImbaposProd is successful in terms of preventing costs from increasing. However, the cost decrease is small, providing only a minor financial incentive for managing the balancing group according to Imba-posProd scenario. For Imba-lexico scenario a significant decrease of costs can be observed, with an optimum of cost reduction for 100 batteries in operation.

From the economic perspective of the balancing group manager, Imba lexico strategy would be chosen, providing the largest economic benefit, and the number of batteries would not be increased above 100. In this case, a decrease of energy imbalances of $17 \%$ of the optimum would be achieved, contributing to smaller deviations in the balancing group and subsequently to smaller fluctuations in the power system.

\section{Impact of BESS size on objectives}

Rated power and capacity of the BESS are limiting factors regarding optimization objectives. The upscaling effects are studied by increasing the number of batteries in this examination, keeping the ratio between rated power and capacity constant. For 150 batteries, the total rated power almost reaches the peak power of the imbalances curve.

Therefore, there is almost no further benefit in increasing power for the imbalance scenarios. Instead, a higher capacity is needed, allowing the balancing over longer timespans. For the cost scenarios, additional batteries would always provide proportional cost decrease.

Yet obviously other limitation will occur in real application, up to a point where the effect of the BESS utilization will significantly influence imbalance prices. For increasing battery number, these effects must be considered to accurately estimate potential benefits of the BESS utilization.

\section{Scenario comparison}

Due to additional restrictions for BESS operation in CostposProd and Imba-posProd scenarios, these cannot provide comparable benefits as the other strategies. For minimizing costs, Cost-simple and Cost-lexico provide the greatest benefit, while the latter perform slightly better in terms of energy imbalance reduction. However, both strategies are substantially increasing imbalances and are therefore critical from the TSO perspective. Imba-simple on the other hand increases cost for balancing group operators and therefore will not be of use. Finally, Imba-lexico reduces both cost and imbalances. Even if cost reduction is smaller compared to Cost-simple and Costlexico, Imba-lexico provides a good tradeoff and serves both objectives.

\section{CONCLUSION AND OUTLOOK}

This paper shows the potential of a BESS utilization for balancing group management. Obviously, if both the cost and energy imbalances need to be reduced, both aspects have to be represented in the optimization strategy. Representation can either be explicit as with the lexicographic method or implicit as for the Cost-posProd and Imba-posProd scenarios. However, representation alone has shown to be insufficient, as prioritizing cost reduction over energy imbalance reduction may lead to creation of additional imbalances. Focus on cost reduction tends to exploit the balancing group management by misusing it for short-term energy trading. Therefore, the 
energy imbalance objective must be prioritized higher than the cost objective.

However, the potential for cost reduction will be the decisive criterion for a balancing group manager for battery utilization, considering the investment and operational costs for this measure. Given these restrictions, potential benefit for battery utilization is limited due to the diminishing marginal benefit a BESS is providing in terms of cost reduction. To estimate the optimal number of batteries in a real application, investment and operational costs of the BESS must also be considered as well as the degree to which the theoretical optimum can be achieved.

In future work, strategies for BESS operation can be investigated, including objective functions including both the cost and energy imbalance aspect. Weighting factors can be utilized to shift the focus between the respective aspects. Additionally, the BESS layout can be further investigated, studying the effects of changing the ratio between rated power and capacitiy.

\section{REFERENCES}

[1] A. Burgio, G. Brusco, D. Menniti, A. Pinnarelli, N. Sorrentino, and P. Vizza, "Economic evaluation in using storage to reduce imbalance costs of renewable sources power plants," in 2017 14th International Conference on the European Energy Market (EEM), Jun. 2017, pp. 1-6.
[2] A. Burgio, D. Menniti, A. Pinnarelli, N. Sorrentino, P. Vizza, and N. Tutkun, "Prosumers with a photovoltaic-battery system: Comparison of two stategies for imbalance reduction," in 2017 IEEE 14th International Conference on Networking, Sensing and Control (ICNSC), May 2017, pp. 19-24.

[3] S. Chakraborty and T. Okabe, "Energy storage scheduling for imbalance reduction of strategically formed balancing groups," in 2016 IEEE International Conference on Smart Grid Communications (SmartGridComm), Nov. 2016, pp. 278-283.

[4] N. G. Paterakis, M. Gibescu, W. Kout, and Y. A. Hugo, "Investigation of arbitrage between the dutch day-ahead and imbalance markets as a business case for the hydrogen bromine flow battery," in 2017 IEEE Manchester PowerTech, Jun. 2017, pp. 1-6.

[5] A. Dammasch and B. Engel, "Reducing the use of compensation energy in a balancing group using a virtual power plant," in International ETGCongress 2013; Symposium 1: Security in Critical Infrastructures Today, Nov. 2013, pp. 1-5.

[6] European Union's Horizon 2020 research and innovation programme (grant agreement No. 646125). Elsa - energy local storage advanced system. [Online]. Available: http://www.elsa-h2020.eu/

[7] Bundesministerium für Wirtschaft und Energie, "Verordnung über den Zugang zu Elektrizitätsversorgungsnetzen,” Jul. 2005.

[8] Allgäuer Überlandwerk $\mathrm{GmbH}$, "Balancing group time series data, energy imbalances and imbalance prices," 2015.

[9] Bundesnetzagentur für Elektrizität, Gas, Telekommunikation, Post und Eisenbahnen, "Bilanzkreisvertrag Konsultationsentwurf," Jun. 2014.

[10] D. Bertsimas and J. N. Tsitsiklis, Introduction to linear optimization, ser. Athena Scientific series in optimization and neural computation. Belmont, Mass: Athena Scientific, 1997.

[11] M. Ehrgott, Multicriteria optimization, 2nd ed. Berlin ; New York: Springer, 2005. 